\title{
THE SOLAR MOTION, THE GALAXY AND THE APHELIA DISTRIBUTION OF LONG PERIOD COMETS
}

\author{
A. BRUNINI \\ Instituto Astronômico e Geofísico, Universidade de São Paulo, Brasil \\ and \\ D.M.CANOSA \\ Facultad de Ciencias Astronomicas y Geofisicas Paseo del Bosque s/n 1900 La Plata \\ Argentina
}

\begin{abstract}
We analyze the incoming direction of 180 long period comets, taken into account the gravitational influence of the planets and the Galaxy as a whole. We have made an improvement of some previous works on the matter, by inclusion of comets observed up to 1989. An asymmetry of aphelia is detected that cannot be explained as due to observational selection. We conclude that the so called Dynamical Friction induced by the solar motion through the interstellar medium could be the proper answer to this observed fact.
\end{abstract}

\section{The data sample}

The comets included in this analysis where taken from Marsden's Cometary Catalogue (version 1990), and were included those comets with periods greater than 10000 years, and with well determined orbits (class A). The whole resultant sample is composed by 180 long-period comets.

To compute the aphelia direction we have taken the orbital osculating elements and performed a backward numerical integration, including all the nine planets, until the comets were at $150 \mathrm{AU}$ from the Sun. At that moment, the planets were removed from the differential equation of motion, and the reference system was changed to galactic one. Then, the galactic tidal field was included (Torbett, 1986). Two situations may occur:

1) The Sun-comet distance become greater than 200000 AU (the limit of stability of the Solar System is about $170000 \mathrm{AU}) 51$ comets among the total sample reached this category, ( and probably some of them have been captured by the Solar System).

2) The Sun-comet distance reach a maximun, where it beginns to decrease. This position was considered the comet aphelium.

\section{Analysis of the aphelia direction}

Perihelic directions of long-period comets, when studied in the equatorial geocentric reference system, present a North-South asymmetry that could be consequence of observational selection mechanisms (see for example Everhart, 1967a and 1967b).

However, the perihelic direction can differ from the direction where the comets were detected by the first time. To examine such a possible selection effect, we have computed geocentric equatorial coordinates of the comets at the moment of the first observation included in the computation of the orbital elements, in the Marsden's catalogue (thereafter: GECD) because they will be good indicators of the place where the comets were discovered. We have plotted these coordinates on 


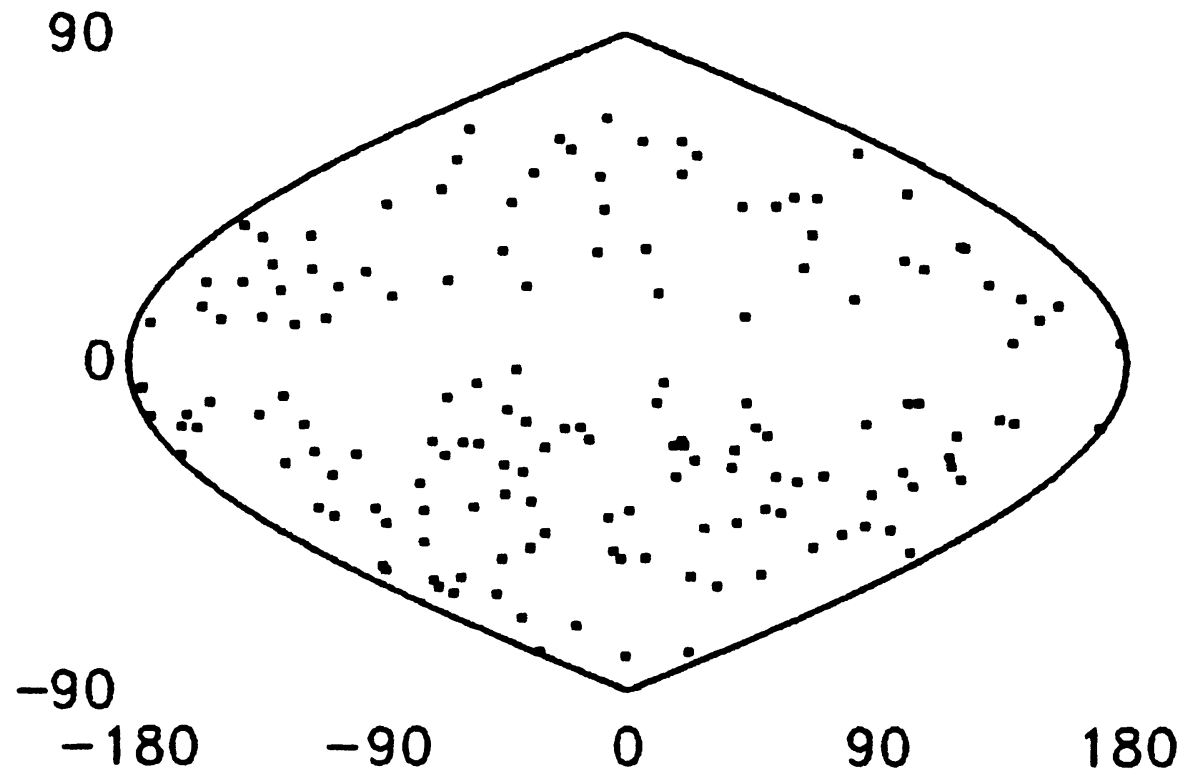

Fig. 1. Aphelia in Galactic coordinates

an equal-area mapping of the celestial sphere, and we have seen a strong NorthSouth asymmetry also mentioned in some previous works.

\section{Distribution in galactic coordinates}

In figure 1 it is shown the distribution of the aphelia in galactic coordinates. A direct visual inspection of this figure shows the two well known cluster of aphelia (not too well separated one from the other).

It is worth noting, however, that both two clusters are located in the hemisphere of the Solar antapex, contributing to an asymmetry already pointed out by some authors (Tyror 1957, Oja 1975). The point now will be to investigate about the nature of this asymmetry. We have annalyzed this particular problem, following the next procedure: we have taken from the whole sample of 180 long period comets, a random sample in such a way that were not found any significative cluster of aphelia. So, we have obtained a new group of 158 comets, whose aphelia distribution in galactic coordinates still presents more number of aphelia in the South galactic hemisphere than in the North one. The new sample, when is analizated in GECD 
presents a near random distribution, except a slight deficiency of comets with aphelia in the South hemisphere and right ascentions between $0 h$ and $12 h$. We do not known the exact origyn of this effect, but in an attemp to investigate if it is due to an observational selection effect, we have tried to compensate the sample by generation of random ficticious comets in order to equilibrate the aphelia distribution in GECD. However, the asymmetry in galactic coordinates is still present, with an excess of about $20 \%$ of aphelia in the South hemisphere.

\section{The role of the solar motion}

In the present section we will look for some dynamical mechanism able to give account of such a particular asymmetric distribution.

An important fact is that, when the sample is arranged in distance, there are an exess of comets in the South hemisphere with aphelia comprised between $1000 \mathrm{AU}$ and $10000 \mathrm{AU}$. These distances repesent an inner zone of the Oort cloud, rather insensitive to stellar passages.

Planet $X$, if it really exists, would be too small to produce any significative perturbation on comets with aphelia beyond $1000 \mathrm{AU}$.

Biermann (1978) first annalized the effects of a solar passage throught a giant molecular cloud, in the context of the impulsive approximation arriving to important results, after corroborating by Bayley (1983), but a high speed passage cannot explain an asymmetry in the direction of the solar motion, because the nature of the impulse approximation itself.

When a star is moving with a low velocity $V$ through a sea of particles assumed at reast (the interstellar medium), this particles fall in hyperbolic orbits by the mutual gravitational action.

Thus, instead the final velocity of the star is in modulus the same that before the encounter, the component in the direction of motion is reduced. If the medium is infinite and homogeneus the change in the component perpendicular to the star velocity is, in average, zero. However, the other component have a systemmatic retardation. This effect was first annalyzed by Chandrashekar (1942) and results in a too important contribution to the study of some observed features in galactic dynamics (See Binney and Tremaine, 1987). This effect was called "dynamical friction" because, in analogy with drag, the star is descelerated, lossing kinetic energy in the work to "deflect particles of the medium".

In this context, the desceleration of the Sun velocity will be almost constant, and in the direction of the Solar antapex.

The comets of the Oort cloud, do not suffer by the same effect, because the mass appears as a factor.

Taking into consideration these facts, the potential of this force field is then

$$
U(r, b)=-G M_{\odot} / r-C r \sin b
$$

Where $b$ is the galactic latitude (more properly, the latitude in an apex system )and $C$ is the constant desceleration of the Sun. 
This potential presents cylindrical symmetry and it is interesting to note that it has an absolute minimun in $b=-90^{\circ}$. So, as in the case of the galactic tidal field the aphelia distribution mimics this dependence with the galactic latitude.

A first order secular analysis of these equations of motion, indicates that this force is able to account for the observed exess of aphelia in the south galactic hemisphere.

\section{Acknowledgements}

We acknoledge the invaluable discussions with Dr. Juan Carlos Muzzio at the La Plata Observatory.

This paper was partially suported by The Sao Paulo Science Foundation (fapesp) and by Fundacion Antorchas.

\section{References}

Bailey M.E.: 1983,Mon. Not. Roy. Astron. Soc. 204, 603.

Biermann L.: 1978, A stronomical Papers dedicated to Bengt Stromgren. eds. Reiz A. and Anderson T. 327-333 Copenhagen University Press.

Binney J. and Tremaine S.: 1987, Galactic Dynamics. Princeton Series in Astrophysics. Princeton University Press.

Chandrasekhar J.: 1942, Principles of Stellar Dynamics. Dover Publications Inc. New York.

Everhart E.:1967a, The Astronomical Journal 72, 718.

Everhart E.:1967b, The Astronomical Journal 72, 1002.

Marsden B.G.: 1990, Catalogue of Cometary Orbits 5th. ed.

Oja, H.: 1975, Astron. Astrophys. 43, 317.

Smith B.A. and Terrile R.J.: 1984, Science 226, 1421.

Torbett, M.: 1986, The Galaxy and the Solar System. eds. R. Smoluchowski, J.N. Bahcall and M.S. Matthews, p.147.

Tyror J.G.: 1957, Mon. Not. Roy. Astron. Soc. 117, 170.

Withmire D.P. Matese J.J and Tomley L.J.: 1988, Astron. Astrophys. 203 L13. 